\title{
THREE-DIMENSIONAL NUMERICAL EXPERIMENTATION ON THE CORE HELIUM FLASH OF LOW-MASS RED GIANTS
}

\author{
David S. P. Dearborn, ${ }^{1}$ John C. Lattanzio, ${ }^{2}$ and Peter P. Eggleton ${ }^{1}$ \\ Received 2005 June 28; accepted 2005 October 31
}

\begin{abstract}
We model the core helium flash in a low-mass red giant using Djehuty, a fully three-dimensional (3D) code. The 3D structures were generated from converged models obtained during the one-dimensional (1D) evolutionary calculation of a $1 M_{\odot}$ star. Independently of which starting point we adopted, we found that after some transient relaxation the 3D model settled down with a briskly convecting He-burning shell that was not very different from what the $1 \mathrm{D}$ model predicted.
\end{abstract}

Subject headings: stars: evolution — stars: interiors

\section{INTRODUCTION}

The core helium flash is an important event in the life of most stars with a zero-age mass between about 1 and $2 M_{\odot}$; the minimum masses are a little lower for metal-poor stars. Since the work of Mestel (1952) and Schwarschild \& Härm (1962) it has been clear that such stars ignite helium in a thermonuclear runaway situation, the helium flash, because the helium core is electron-degenerate at the time of ignition. Empirically, it is clear that this runaway is (usually) not a catastrophic affair, like a supernova explosion, because a whole class of stars, the horizontal branch stars of globular clusters, is well explained by the survival of helium flash stars in a long-lived state of core helium burning (Faulkner 1966). Nevertheless, attempts to compute the evolution during the flash have a confusing history: some calculations (both 1D and 2D) have predicted a rather severe explosion, and others (both 1D and 2D) a relatively benign, although rapid, ignition.

Most calculations until fairly recently have been 1D simulations, in which turbulent convection has been treated by a spherical averaging process based largely on the mixing length concept. Deupree (1996), who gives a nice summary of earlier work, performed some 2D (axially symmetric) simulations. He found that the $2 \mathrm{D}$ estimates were critically dependent on approximations made regarding eddy viscosity. We expect that by working in 3D we will not need to make such approximations, and we suggest that our results bear out this expectation.

The Djehuty project of the Lawrence Livermore National Laboratory is an effort to model stars in 3D. Our ultimate aim is to be able to model an entire star, up to and including the photosphere and, indeed, to generalize this to binary stars, including gas flows between them in, for example, a Roche lobe overflow situation. We are approaching this goal, but it is fairly easy to see that a star such as the Sun, for instance, would require at least $10^{12}$ nodes inside it if there is to be adequate resolution near the surface. As computer power continues to increase, this will no doubt become possible, but for the present we limit ourselves to about $10^{8}$ nodes. We therefore content ourselves with a

\footnotetext{
${ }^{1}$ Lawrence Livermore National Laboratory, 7000 East Avenue, Livermore, CA 94551; dearborn2@1ln1.gov, ppe@igpp.uclln1.org.

${ }^{2}$ Department of Mathematics, Monash University, Clayton, Victoria 3168, Australia; john.lattanzio@sci.monash.edu.au.
}

simulation of the He flash that includes only the He core and the radiative portion of the envelope; we ignore the deep surface convection zone.

Apart from the intrinsic interest of the He flash, our other reason for pursuing this particular problem is that it potentially is a very good test for the stability and accuracy of a hydrodynamic code. This is because we have, as mentioned above, a rather good reason to anticipate what the outcome should be. We do not expect it to become a violent supernova-like event. In following an explosion, it is not easy to look at the outcome and say "that is clearly what should have been expected," even if it is what we expected. But in a nonexplosive situation it is not difficult to compare, for instance, the heat flux actually carried by turbulent convection across a spherical shell with the expectation from a simple mixing length model.

In this paper we consider only nonrotating and nonmagnetic cores, but we believe that both these processes could be important, and we hope to address them in a later paper. In $\S 2$ we briefly outline the code; in $\S 3$ we describe our 1D input models and 3D output models. In $\S 4$ we describe some issues and numerical tests regarding the stability of the calculation.

\section{CODE DESCRIPTION}

Djehuty is a code designed to model entire stars in 3D. Developed at the Lawrence Livermore National Laboratory (LLNL), it operates in a massively parallel environment and includes the basic physics necessary for modeling whole stars. Earlier descriptions of the Djehuty code can be found in Bazán et al. (2003) and Dearborn et al. (2005), but a brief description is provided here.

For a star, the Djehuty mesh is formed from seven logically connected blocks of hexahedral cells of variable shape. There is a central cube of $N \times N \times N$ cells, surrounded by six logical cuboids $(N \times N \times L)$. One of the two $N \times N$ faces of each cuboid is attached point by point to the face of the central cube. The other $N \times N$ face of each cuboid is mapped to lie on a spherical surface forming the outer boundary. The cuboids are then "morphed" into wedge shapes with surfaces on the long $(L)$ radial axis, transitioning from planar to spherical. The $N \times L$ faces are similarly attached to adjacent cuboids in a point-by-point fashion. An exploded version, with the logical structure of the central cube and surrounding cuboids, is shown in Figure 1 (top) with $N=50$ and $L=100$; Figure 1 (bottom) shows two of the blocks 

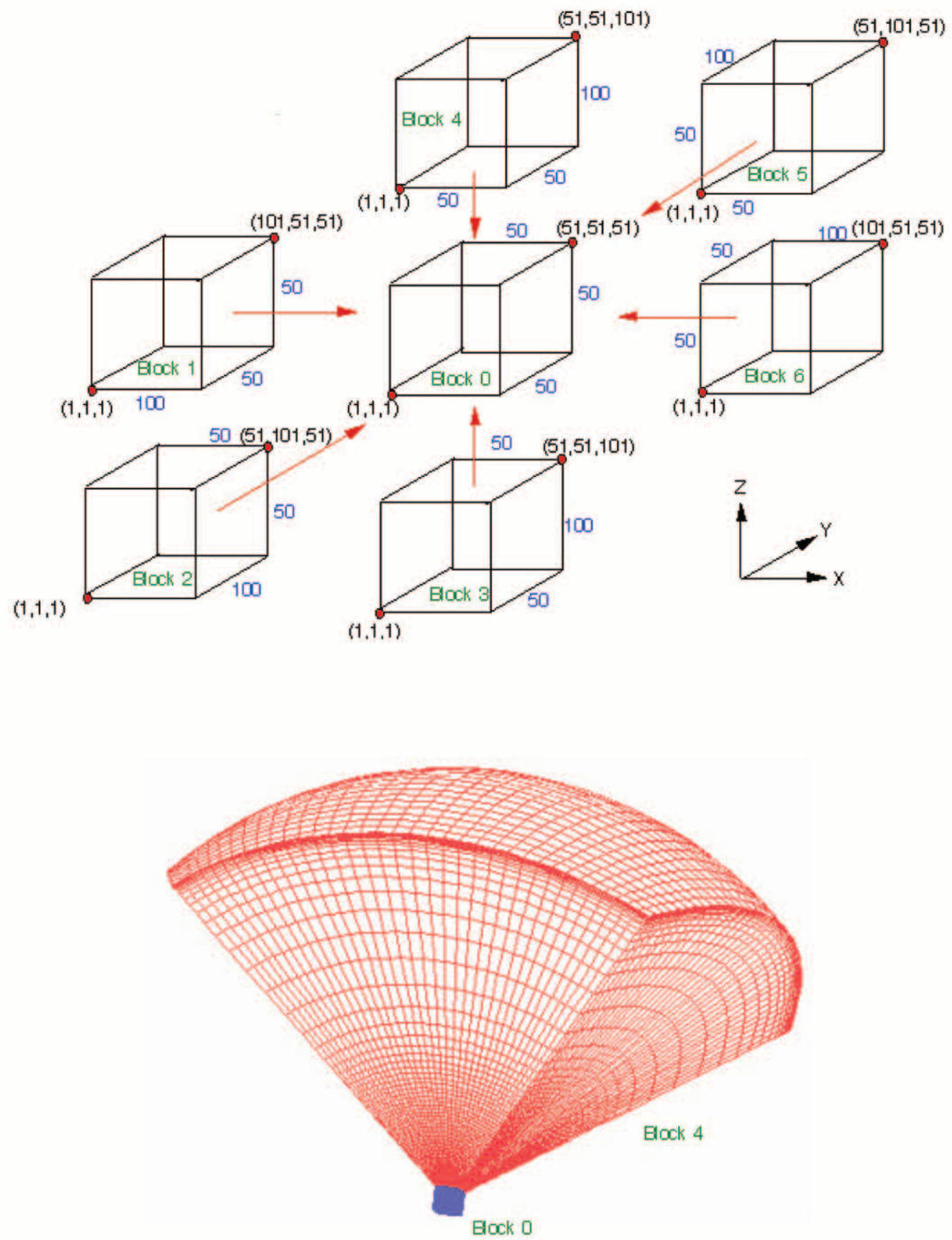

Fig. 1. - Top, Exploded view of the mesh structure, in logical space; bottom, blocks 0 and 4 in coordinate space (here $N=35$ and $L=70$ ). Block 0 is a great deal smaller than block 4 .

connected together and in physical space, with $N=35$ and $L=70$

This mesh structure allows reasonable azimuthal resolution without the core convergence problem (tiny zones and tiny time steps at the center) endemic in spherical coordinate systems. In the core itself, the cells are nearly rectangular, and as the radius grows, successive surfaces become more spherical, matching the potential surfaces, as well as properties such as temperature and pressure. The mesh can encompass an entire star with free outer boundaries, as was done by Dearborn et al. (2005), or a portion of the star with various fixed boundary conditions, as is done here. For reasons discussed later, in these calculations we locate the outer boundary at the inner edge of the red giant's deep convective envelope.

The initial 3D structures are generated from models produced by a 1D hydrostatic stellar evolution code. This 1D code was used as a platform to test the physics (equation of state, nucleosynthesis, etc.) incorporated into Djehuty, as well as to provide structure information for constructing 3D models. Djehuty reads the $1 \mathrm{D}$ stellar models, mapping the defining physical parameters on to a 3D spherical grid at any given stage of evolution. The radial structure of the $1 \mathrm{D}$ mesh is used as a guide in scaling the 3D mesh, so that regions with steep gradients, such as thin burning shells, are resolved. Scalar quantities such as 
pressure, temperature, and composition are assigned to the cell centers, and vector quantities (position and velocity) are assigned to nodes. The position of the cell center is the direct average of the eight radius values of the nodes surrounding it.

The 1D code carries six elements $\left({ }^{1} \mathrm{H},{ }^{3} \mathrm{He},{ }^{4} \mathrm{He},{ }^{12} \mathrm{C},{ }^{14} \mathrm{~N}\right.$, and ${ }^{16} \mathrm{O}$ ) directly, with the remainder assumed to be ${ }^{24} \mathrm{Mg}$. This set of elements was selected to allow accurate tracking of the principal energy-generation reactions for hydrogen and helium burning over a broad range of masses and evolutionary stages. The seven-element set includes the triple-alpha reaction, as well as the alpha captures on carbon and nitrogen, to provide the energy production, but the $1 \mathrm{D}$ code does not follow ${ }^{18} \mathrm{O}$, so that the capture on nitrogen is assumed to be a $2.5 \alpha$ capture to the heavyelement remainder $\left({ }^{24} \mathrm{Mg}\right)$.

The $3 \mathrm{D}$ code is capable of operating with the same element set as the $1 \mathrm{D}$ code, but for this calculation the $3 \mathrm{D}$ code followed a 21 element set for a more accurate definition of the helium-burning results. The elements followed are ${ }^{1} \mathrm{H},{ }^{3} \mathrm{He},{ }^{4} \mathrm{He},{ }^{12} \mathrm{C},{ }^{13} \mathrm{C},{ }^{13} \mathrm{~N}$, ${ }^{14} \mathrm{~N},{ }^{15} \mathrm{~N},{ }^{15} \mathrm{O},{ }^{16} \mathrm{O},{ }^{17} \mathrm{O},{ }^{18} \mathrm{O},{ }^{17} \mathrm{~F},{ }^{18} \mathrm{~F},{ }^{19} \mathrm{~F},{ }^{20} \mathrm{Ne},{ }^{22} \mathrm{Ne},{ }^{24} \mathrm{Mg},{ }^{28} \mathrm{Si}$, ${ }^{32} \mathrm{~S}$, and ${ }^{56} \mathrm{Ni}$. In the seven-element mode, the rate equations are identical in the $1 \mathrm{D}$ and $3 \mathrm{D}$ codes. When the 21-element set is used, the $3 \mathrm{D}$ code has those rates, as well as additional rates and couplings. The 21-element set is connected with an extensive set of nuclear reaction rates including hydrogen-, helium-, carbon-, and oxygen-burning reactions, as well as a small NSE (nuclear statistical equilibrium) approximation ( Timmes et al. 2000). Tests using the two networks for a range of fixed conditions representative of static hydrogen and helium burning have shown that the networks are well matched in their energy production rates. Coulomb screening (Graboske et al. 1973) and neutrino cooling (Itoh et al. 1989, 1992, with errata) are implemented in both codes.

The 1D code uses the Eggleton (1971) approach for implicit, adaptive mesh adjustment, with simultaneous implicit solution for the mesh and the composition along with the structure, permitting the code to continue smoothly and efficiently through radical structural adjustments. The differencing is done in such a way as to permit a solution by the technique pioneered in the stellar structure context, and for the structure variables only, by Henyey et al. (1959), in which some boundary conditions are central and some are at the surface.

In $1 \mathrm{D}$, convection is always modeled by an approximate process. In our $1 \mathrm{D}$ code, convective energy transport is treated with a standard mixing length approach, and element mixing is modeled as a diffusion process using a second-order differential equation with advection and nuclear-burning terms for each element. Limits of convective regions are determined with a Schwarzschild stability criterion.

The 3D code has no such approximations. Material moves when there is a force. We have striven for sufficient spatial resolution to ensure that the larger convective eddies that arise spontaneously are reasonably resolved. It is these larger eddies that tend to carry most of the heat. The code provides options for "subgrid modeling," i.e., estimates of how much heat might be carried by unresolved small-scale eddies, but we did not use them. Our mesh allowed 10-20 cells (in each dimension) within the major eddies, and we feel that this is sufficient for their resolution. This feeling is supported by comparing runs with a mesh that had twice as many cells. The energy transport, as indicated by the helium-burning rate, was effectively the same. While the resolution used here appears sufficient for modeling the physical behavior of interest (stability of the helium-burning shell), we do not claim to have captured the smaller scale eddies through which the kinetic energy of the turbulent flow actually merges into the thermal field.
Beyond the modest resolution, the small-scale end of the turbulence spectrum is terminated by the ALE (arbitrary LagrangeEulerian) hydrodynamic method, used to allow a Lagrangian representation to survive in a sheared region. When motion causes the Lagrangian mesh to be sufficiently distorted, an Eulerian remap step, or, more precisely, an interpolation step performed in a manner similar to what is used in a numerical approximation to advection, can mix the composition of adjacent cells while smoothing the mesh structure. In stable regions, or regions of large-scale coherent motion where the Lagrangian mesh is modestly deformed, no remapping is necessary, and the code is essentially Lagrangian. In regions where shear develops, it is necessary to relax the mesh and permit material to move between cells. The transition to an Eulerian result is smooth and accurate.

Such a code incorporates, in effect, two kinds of artificial viscosity. Any finite-difference scheme, Lagrangian or Eulerian, implies artificial viscosity through approximating derivatives as finite differences. In addition, the remap step that is applied from time to time to prevent major distortion of the (normally) Lagrangian nodes also introduces a form of numerical diffusion or viscosity. We rely on these, and these alone, to prevent the creation of smallscale (unresolvable) eddies, which would normally be driven by the spectrum of eddies that are resolved, and that are liable to destroy the calculation if unchecked.

The ALE scheme implemented here was tested by Anderson et al. (2004), who ran a number of standard hydrodynamics tests comparing it to an Eulerian high-order Godunov scheme. They found that the accuracy of the two schemes was generally equivalent and that the ALE scheme was much improved over an earlier comparison reported by Woodward \& Colella (1984). A predictor-corrector formalism promotes hydrodynamic accuracy and time centering for other physical processes. The hydrodynamics step is second-order accurate in both time and space.

Both the 1D and 3D codes use the same analytic equation of state developed by Eggleton et al. (1973), as updated by Pols et al. (1995), which provides continuous thermodynamic derivatives for hydrodynamic consistency. It includes molecular hydrogen binding, as well as ionization of the light elements, and can reproduce tabulated values of Rogers \& Iglesias (1992) to much better than $1 \%$ accuracy for the entire range of conditions expected in stars between 0.7 and $50.0 M_{\odot}$ over their whole evolution.

The code operates with separate matter and radiation temperatures integrated in flux-limited diffusion equations. These equations include energy source and sink terms that link the radiation equation to the hydrodynamic energy and momentum equations, as well as to nuclear energy production and neutrino losses. Planck and Rosseland mean opacities are derived from the OPAL library at LLNL and Alexander opacities for the lower temperatures (Alexander \& Ferguson 1994; Rogers \& Iglesias 1992). Conduction coefficients for electron heat flow are from the conductive opacities tables of Hubbard \& Lampe (1969), as modified by Itoh et al. (1983).

The hydrodynamics and the gravitational potential are purely Newtonian; the latter uses integration of a mass-radius relation to generate a spherical potential that approximates the gravity. While this is adequately accurate for the problem considered here, we are developing a multipole approximation to the gravitational potential for use in less spherical systems.

As a final note on the 1D modeling of a helium flash, the conditions make numerical stability a challenging problem. To this end, the 1D code was made fully (64 bit) double precision, the derivatives necessary for this solution were all done analytically, and the convergence criteria for the Newton-Raphson 
solver were set tightly (usually 1 part in $10^{7}$, although 1 part in $10^{6}$ seemed adequate in relatively easy phases).

\section{THE HELIUM FLASH}

\subsection{One Dimension}

Each of the 3D simulations done here started from a basic model generated as part of the evolutionary sequence of a $1 M_{\odot}$ Population I star. It assumed an initial hydrogen mass fraction of 0.7 and metals abundance $(Z)$ of 0.02 . The model had 750 zones and a mixing length ratio of 1.8 . With these initial conditions, it did not match our best solar model, but was close $\left(L=1 L_{\odot}\right.$ at 4.58 Gyr and $\left.R=1.02 R_{\odot}\right)$.

Modeling began with a fully convective pre-main-sequence structure having no nuclear reactions. The $1 \mathrm{D}$ evolution passed through the main sequence and the giant branch to the helium core flash and, finally, to core helium burning (Fig. 2). The helium core flash occurs at the tip of the giant branch, when the model is 12.2 Gyr old and has a radius of $175 R_{\odot}$. At this point the core mass is $0.472 M_{\odot}$, with a radius of $0.026 R_{\odot}$. Before helium ignition all of the $2670 L_{\odot}$ is derived from hydrogen burning in a thin shell. Surrounding the hydrogen-burning shell is a radiative region, which contains only about $0.002 M_{\odot}$, but whose radial extent exceeds $\sim 1 R_{\odot}$, or 40 core radii. Over this radiative region, the density drops by nearly 5 orders of magnitude.

During the entire 1D evolution mass loss by stellar wind was not included. It is well known that some mass loss, probably before the helium flash, as well as after it, is to be expected. However, it is also well known that the helium core on the approach to the helium flash is rather little affected, provided only that the mass loss does not strip the star right down to the core. Since the core at the helium flash was $0.472 M_{\odot}$, the star would have had to lose somewhat more than half its mass. This is not

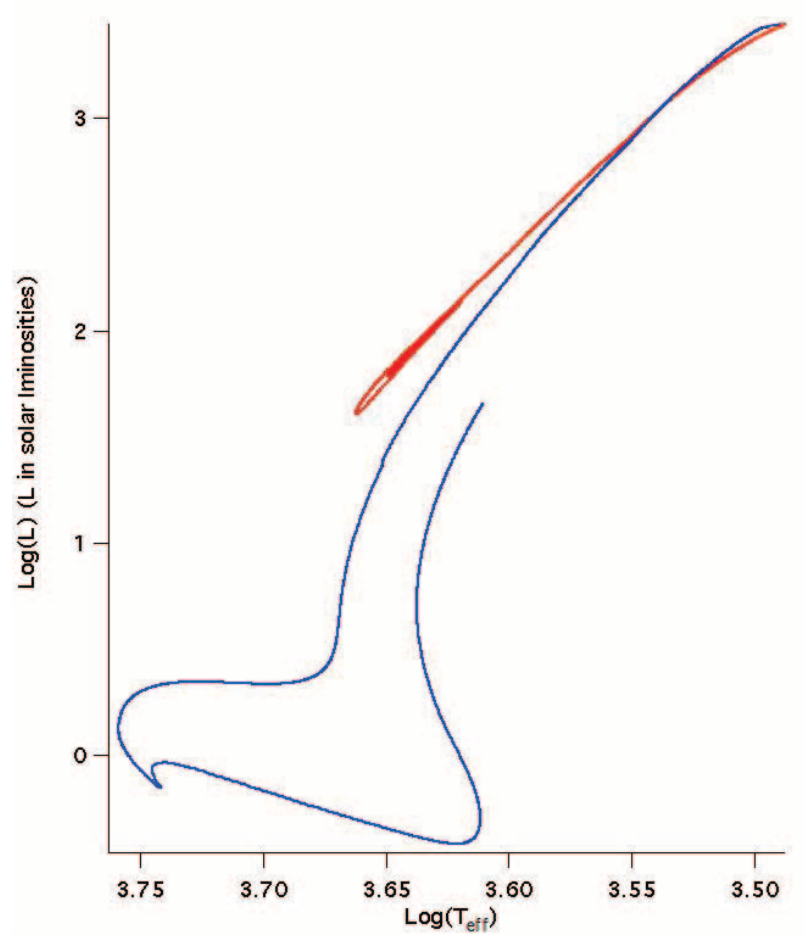

FIG. 2.-1D evolution of our $1 M_{\odot}$ model in the theoretical HRD. Preflash evolution is in blue, and postflash evolution is in red. During the later stage of evolution to the horizontal branch there were two major oscillations (miniflashes), which appear as a somewhat broadened part of the red track.
TABLE 1

The Time Difference $t-t_{\text {peak }}$ (DAYs)

\begin{tabular}{|c|c|c|}
\hline $\log \left(L_{\mathrm{He}} / L_{\odot}\right)$ & Prepeak & Postpeak \\
\hline$\ldots \ldots \ldots$ & -3.28 & 4.38 \\
\hline $8 \ldots$ & -14.23 & 28.47 \\
\hline $7 \ldots$. & -67.16 & 202.57 \\
\hline 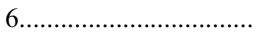 & -375.95 & $1.5 \times 10^{3}$ \\
\hline 5 & $-2.2 \times 10^{3}$ & $1.2 \times 10^{4}$ \\
\hline 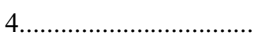 & $-1.4 \times 10^{4}$ & $9.8 \times 10^{4}$ \\
\hline $3 \ldots \ldots$. & $-7.0 \times 10^{4}$ & $5.2 \times 10^{5}$ \\
\hline 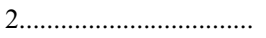 & $-4.9 \times 10^{5}$ & $1.3 \times 10^{6}$ \\
\hline
\end{tabular}

out of the question, but it is probably an estimate on the high side. If either (1) the star lost only $0.45 M_{\odot}$ or, alternatively, (2) the star started at, say, $1.3 M_{\odot}$ and lost $0.75 M_{\odot}$, the core at the flash would be very much the same as the one we obtained. Thus, we feel that the "default option" of no mass loss is a very reasonable simplification.

In the helium core, neutrino cooling reduces the central temperature, shifting the initial helium-burning region off-center. Energy production from helium burning starts in a narrow region at about $0.18 M_{\odot}$, or $0.008 R_{\odot}$, from the center. As the energy production rate from helium burning becomes significant, a convective shell is developed to transport the energy. The 1D hydrostatic code uses a mixing length approach that has no time dependence for the start-up of convective heat transport (although, because mixing of composition is modeled by a diffusion process, the response of composition is not instantaneous). As is the common practice, the $1 \mathrm{D}$ code uses a stability criterion to determine where convection is necessary. As we show in our 3D modeling, the timescale for developing convection, as well as its efficiency at removing energy from the very thin burning region, is critical to the stability of the simulations, as well as to the timescale for the flash.

While the ignition of helium is fast by the standards of stellar evolution, it is slow compared to hydrodynamical timescales (the sound travel time across the core, a few seconds). From the time that the energy production rate from helium burning reaches $100 L_{\odot}$, it takes about $1000 \mathrm{yr}$ to reach $1000 L_{\odot}$, and another $140 \mathrm{yr}$ to reach $10^{4} L_{\odot}$. The energy production rate peaks above $3 \times 10^{9} L_{\odot}$ for a period just over $30 \mathrm{hr}$, and the model spends about 7 days producing energy above $10^{9} L_{\odot}$ (Table 1 ). During the height of the energy production, nearly all of this energy is used to expand the outer portion of the core (Fig. 3), changing its thermal structure. As the core expands, the energy produced by hydrogen burning at the edge of the core falls. By the time of peak energy production, hydrogen burning produces only about $200 L_{\odot}$. It is only $0.03 L_{\odot}$ a year after the peak of the helium flash. About $8000 \mathrm{yr}$ after the peak of the helium core flash, the hydrogen-burning shell has begun to reestablish itself at a lower level, and the energy production rate by both hydrogen and helium burning is near $20 L_{\odot}$.

Because the energy produced by the hydrogen-burning shell drops, it is unable to support the very large radius. As the energy stored in the envelope leaks out, the radius decreases. In the $15,000 \mathrm{yr}$ following the peak of the helium flash, the radius drops to about $20 R_{\odot}$, near the value that will be supported by core helium burning.

The initial burst of helium-burning energy is absorbed in a convective shell and overexpands the core. This quenches the helium burning (Fig. 4), and the convective shell stabilizes. At this point, the core structure includes a central region of about 


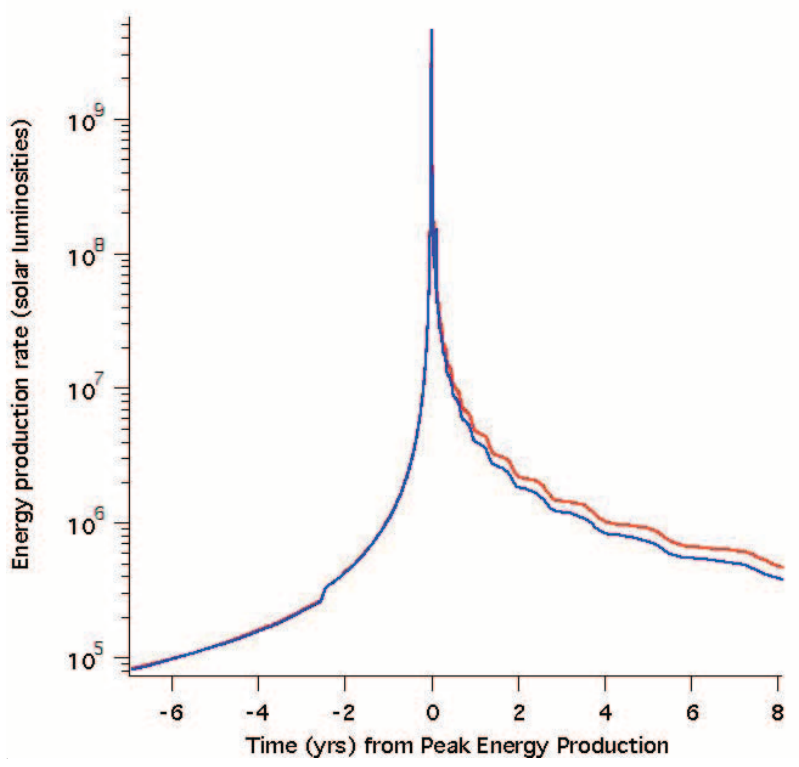

FIG. 3.- In the 1D model, the time history of the energy production rate (in $L_{\odot}$ ) from helium burning is shown in red. The absolute value of the rate of change in the thermal energy of the model is shown in blue.

$0.16 M_{\odot}\left(0.008 R_{\odot}\right)$, where no helium burning has occurred (yet), surrounded by a thoroughly mixed shell region.

Eventually, the model radiates the excess thermal energy that it has absorbed and attempts to reestablish helium burning. Following this initial flash, the mass fraction of ${ }^{12} \mathrm{C}$ averaged over the entire core is 0.025 . On a timescale of about $2 \times 10^{5} \mathrm{yr}$, the model experiences two more mini-helium flashes. These miniflashes appear in the H-R diagram (HRD; Fig. 2) as a couple of narrow loops near the ultimate core helium-burning luminosity. Following the second miniflash, the fluctuations are small enough that the associated convective region is never extinguished. Ultimately, about $2 \times 10^{6} \mathrm{yr}$ after the peak, the center becomes completely mixed, and the average mass fraction of ${ }^{12} \mathrm{C}$ is 0.035 .

\subsection{Three Dimensions}

The entire helium flash duration is much too long to be followed by a Courant-limited hydrodynamics code, but such a code is essential to study the timescale on which convection develops to stabilize the nuclear burning. This development is

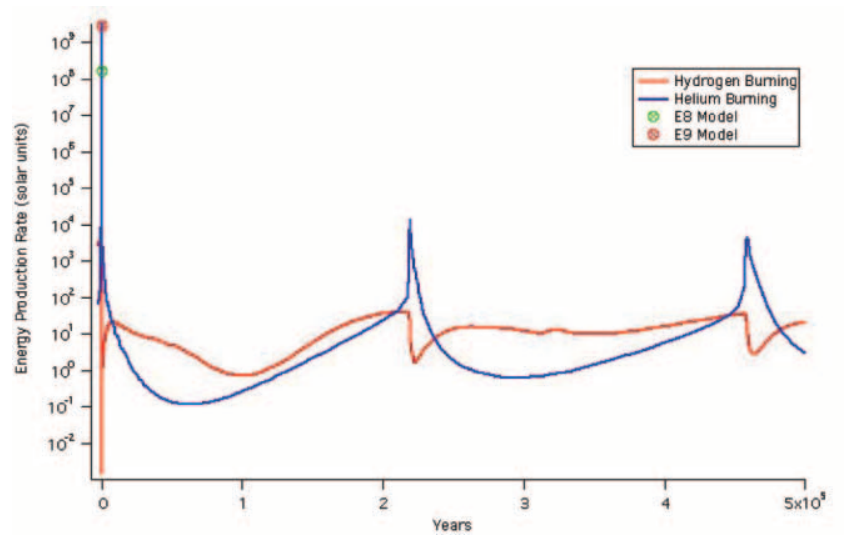

FIG. 4.- In the 1D model, following the initial helium flash, the two subsequent miniflashes as the core relaxes to a fully convective structure.
TABLE 2

Parameters for the Four Simulations

\begin{tabular}{|c|c|c|c|c|c|}
\hline \multicolumn{4}{|c|}{ Mesh } & \multirow{2}{*}{$\begin{array}{l}\text { Run TIME } \\
\text { (s) }\end{array}$} & \multirow{2}{*}{$\begin{array}{r}R_{\text {fixed }} \\
(\mathrm{cm})\end{array}$} \\
\hline Model & $L_{\mathrm{He}}$ & Megazones & Processors & & \\
\hline $\mathrm{E} 4 \ldots \ldots$ & $10^{4}$ & 0.39 & 24 & 4694 & $6.0 \times 10^{10}$ \\
\hline E8 ...... & $2 \times 10^{8}$ & 0.39 & 24 & 6605 & $6.5 \times 10^{10}$ \\
\hline E9 ..... & $3 \times 10^{9}$ & 0.86 & 31 & 3665 & $8.2 \times 10^{10}$ \\
\hline $\mathrm{E} 9^{\prime} \ldots$ & $3 \times 10^{9}$ & 1.32 & 62 & 1917 & $8.2 \times 10^{10}$ \\
\hline
\end{tabular}

the fundamental determinant for the duration of the flash and the type of star that emerges from it. Modeling in 3D permits a direct simulation of the convection process, as well as numerical testing of the sensitivity to perturbations or limitations (such as resolution).

For this study, 1D models were stored at intervals along the evolutionary track, and three of them were selected to study in four 3D simulations ( Table 2). As noted in the code description, Djehuty is capable of modeling entire stars, but for this problem, it is the helium core and hydrogen shell region that are of interest. It contains nearly half of the mass of the star, but only $10^{-12}$ the volume. The vast bulk of the star is in the convective envelope. To concentrate our effort on the portion of the star that was pertinent to this study, we modeled only a portion of the star, selecting a radius that is in the radiative region below the convective envelope. This outer radius was held fixed in temperature and size (Fig. 5). Even here we were modeling a region that had nearly 30 times the radius (27,000 times the volume) of the helium core, and across which the density drops by nearly 5 orders of magnitude.

One of the three models, E4, was selected to be a relatively benign case, with an energy production rate from helium burning near $10^{4} L_{\odot}$. This model represents the state of the star more than half a century prior to the peak energy production rate, and less than $10^{-5}$ of that rate. The other two models were chosen to be near (E8) or at (E9) the peak energy production rate. Information on the selected models is given in Table 2. All the 1D starting models are available electronically, on request.

In doing this suite of simulations we tried various resolutions (meshes with $0.39,0.86$, and 1.32 million zones) and tested different approaches to settling the transients associated with imperfections in the 1D-to-3D mapping process. Despite the efforts to match the $1 \mathrm{D}$ and $3 \mathrm{D}$ codes, the mapping process results in small deviations from hydrostatic equilibrium. While the resulting motion should decay in the fullness of time, stellar interiors are remarkably good oscillators, and the motion can persist for quite a long time. In effect, the lack of exact hydrodynamic equilibrium in the initial discretized model creates artificial pressure waves that can bounce around the model for many crossing times. The situation is made worse by the fact that as waves move toward the surface, down the considerable density gradient, they can reach the force of a tsunami.

Among the settling options is one (Zerovel) that is simply to set to zero the accumulated velocities, usually in outer regions, where the wave has gathered strength. A less intrusive option is to introduce, temporarily, a velocity limiter $\left(V_{0}^{\prime}\right)$. This speed limiter is a very severe kind of artificial viscosity, as follows. Suppose that the acceleration computed at a particular node is $\boldsymbol{a}$. Then the equation of motion is taken to be

$$
\frac{D \boldsymbol{v}}{D t}=\boldsymbol{a} \text { if }|\boldsymbol{v}| \leq V_{0}^{\prime},
$$



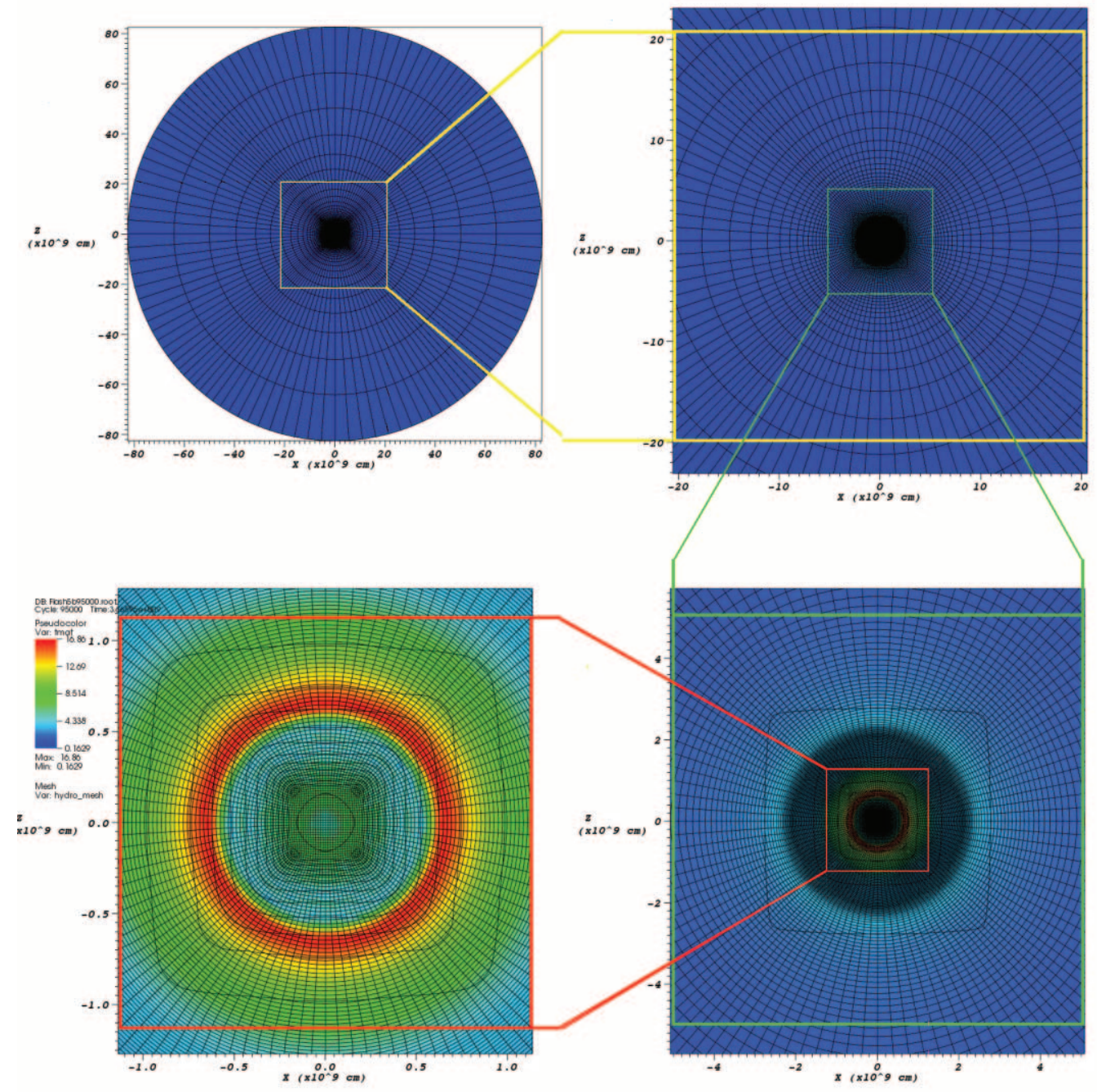

Fig. 5.-For model E4, the mesh, shown on a plane slice through the star, slightly off center. The color background is scaled to the temperature, blue for cold and red for hot. The peak temperature is about $17 \mathrm{keV}$. The high temperature ring in the bottom left panel is the base of the helium-burning shell. The central mesh block, block 0 , is contained inside this region. In the lower two panels, some closed curves that form squarish figures with rounded corners are artifacts of the visualization code, when it is required to visualize the mesh rather than a variable such as temperature. They arise because a plane surface cuts through the sphere, and this surface does not, as a rule, cut exactly through any of the mesh points. Neighboring mesh points and lines are projected on to it, but where the nearest mesh points happen to be equidistant from the surface on opposite sides this artifacts is produced. It is a kind of interference pattern.

but

$$
\frac{D \boldsymbol{v}}{D t}=\boldsymbol{a}-\boldsymbol{v} \frac{|\boldsymbol{v}|-V_{0}^{\prime}}{\delta t|\boldsymbol{v}|} \text { if }|\boldsymbol{v}| \geq V_{0}^{\prime}
$$

where $\delta t$ is the time step and $V_{0}^{\prime}$ is a critical ("treacle") speed.

The speed limiter has been implemented in two forms. In the form used here, $V_{0}^{\prime}$ is a user-specified constant, but it is also possible to select an option in which it scales with the node mass,

$$
V_{0}^{\prime}=V_{0}\left(\frac{\delta M}{\delta M_{0}}\right)^{1 / 2}
$$

where $\delta M$ is the mass of a cell, $\delta M_{0}$ is the largest cell mass in the star (usually near the center), and $V_{0}$ is a specified value. Either option effectively prevents the velocity from becoming much larger than $V_{0}^{\prime}$, in a smooth manner that does not lead to near-discontinuities.

The constant $V_{0}^{\prime}$ option was used here, because the drop in density, although large, is not as large as in a whole star, where the variable option is currently being tested. The constant option provides a well-defined user constraint that minimizes its impact. In the second option, the dependence on $\delta M$ makes the damping more vigorous near the surface, where $\delta M$ is small, and prevents the artificial pressure waves from becoming large toward the outer layers. After some lapse of time, determined by trial and error, $V_{0}^{\prime}$ is removed, i.e., effectively raised to infinity, so that the correct equation of motion is solved from then on.

During the settling-down process we expect pressure waves to radiate more or less spherically from the core. Given that we have an artificially fixed outer boundary, we might expect them to bounce back and cause further trouble. However, the treacle viscosity, which we described above, seems to be effective at 

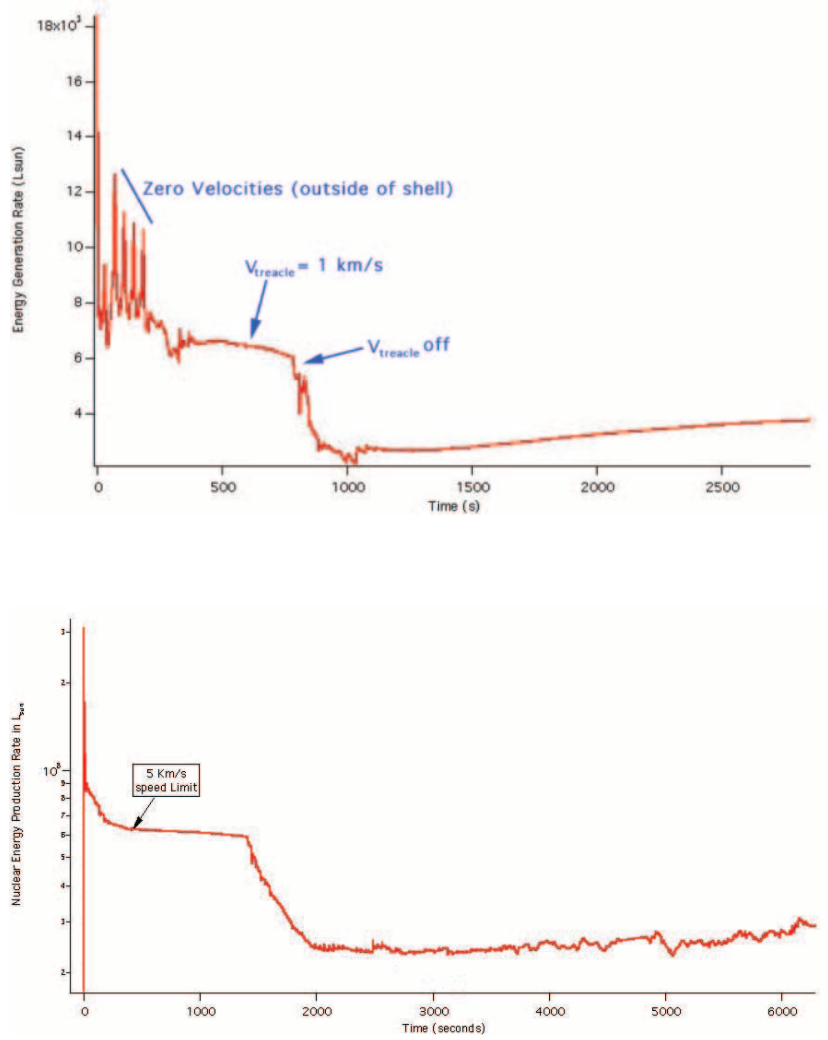

FIG. 6.-Top: Model E4, showing the impact of using Zerovel, as well as a constraint on the speed. Bottom: Model E8, in which Zerovel was not used Here a velocity limiter of $5 \mathrm{~km} \mathrm{~s}^{-1}$ allowed rapid settling from any imbalance from hydrostatic equilibrium. In both cases, the speed limiter stabilized the energy production rate at a higher value than occurred without a limit.

damping them to insignificance even before they reach the outer boundary.

For the helium flash problem, this settling option also provides an opportunity to perform numerical experiments on the sensitivity to convection. Setting it to a value for which only the very fastest nodes were affected allowed a quantitative assessment of the importance of convective efficiency. The result of such a numerical experiment is shown in Figure 6, plotting the time history of the energy production rates of models E4 and E8. Both simulations show an initial spike in energy production associated with the lack of velocity information for the initial model. Without convective motion, the temperature of material in the helium ignition region climbs swiftly. In model E4, the energy production rate reaches $1.8 \times 10^{4} L_{\odot}$ after only $0.3 \mathrm{~s}$. This layer rapidly expands, becoming Rayleigh-Taylor unstable, and initiates convection. As buoyant fingers of material begin to rise and are replaced by cooler material from above, the temperature is stabilized, and the energy production rate returns to the expected value. Similarly, in model E8, there is an initial spike to $3 \times 10^{8} L_{\odot}$, and with the onset of convection the rate returns to the expected value after about $20 \mathrm{~s}$.

For our first 3D simulation, E4, we experimented with the Zerovel approach to settling the initial structure. In the first few hundred seconds we attempted a series of Zerovel tests in which the velocities outside the convective shell were set to zero. Each of these perturbations resulted in a small energy spike, and the effort was abandoned. During this time, out to approximately $800 \mathrm{~s}$, the speed limiter was set to $1 \mathrm{~km} \mathrm{~s}^{-1}$. Over this period, the energy production rate stabilized at about $6000 L_{\odot}$. When we removed the speed limit entirely, convection near the hottest spots could operate at a higher speed, and the energy production from helium burning dropped to about $3000 L_{\odot}$. Subsequently, it increased smoothly to $4000 L_{\odot}$ after about an hour. A stable but very thin convective shell had developed, which was within the region predicted by the $1 \mathrm{D}$ code. The energy production rate was slightly lower, but appeared to be approaching the original 1D model.

With the experience gathered from modeling E4, we moved to simulate a much more energetic structure, E8. This model represents a star less than 15 days prepeak, with an energy production rate from helium burning that is just over $10^{8} L_{\odot}$. The velocity limiter was set to provide a speed limit near $5 \mathrm{~km} \mathrm{~s}^{-1}$ in the core, and we left it active for about $1400 \mathrm{~s}$ after start-up. At the imposed speed limit this was time for nearly two complete turnovers of the convective shell, although relatively little material was actually moving fast enough to activate the limiter. The result of this speed limit was a convective shell that had a stable energy production rate of about $7 \times 10^{7} L_{\odot}$. The speed limiter significantly impacted the energy production rate, because the high-velocity nodes were systematically associated with the warm spots, where the bulk of the energy production occurs.

After $1400 \mathrm{~s}$, the speed limit was repealed, and in less than a turnover time, the luminosity dropped to about $3 \times 10^{7} L_{\odot}$. The model was then followed for an additional $4600 \mathrm{~s}$, sufficient for many convective element turnovers. The final energy production rate here was about a factor of 3 below the 1D hydrostatic model value. This is the largest difference seen between the 1D and 3D models. Given the tremendous sensitivity of the energy production rate to the precise structure, we took the result to be acceptable, but it is certain that the long-term application of the velocity limiter has the effect of overproducing energy and expanding the structure. It is possible that the forced structure change led to the lower final luminosity.

The next model E9 considered in 3D represented the state of the star very near the peak of the energy production rate, near $3 \times 10^{9} L_{\odot}$. The $1 \mathrm{D}$ model spends about $31 \mathrm{hr}$ at this high-energy production rate before the rate begins to decrease. The composition structures for the $10^{8} L_{\odot}$ model and this one are shown in Figure 7. Over the intervening days between these models, the whole core (as defined by the hydrogen-burning shell) has expanded with an average speed of $2.5 \mathrm{~m} \mathrm{~s}^{-1}$, and the helium

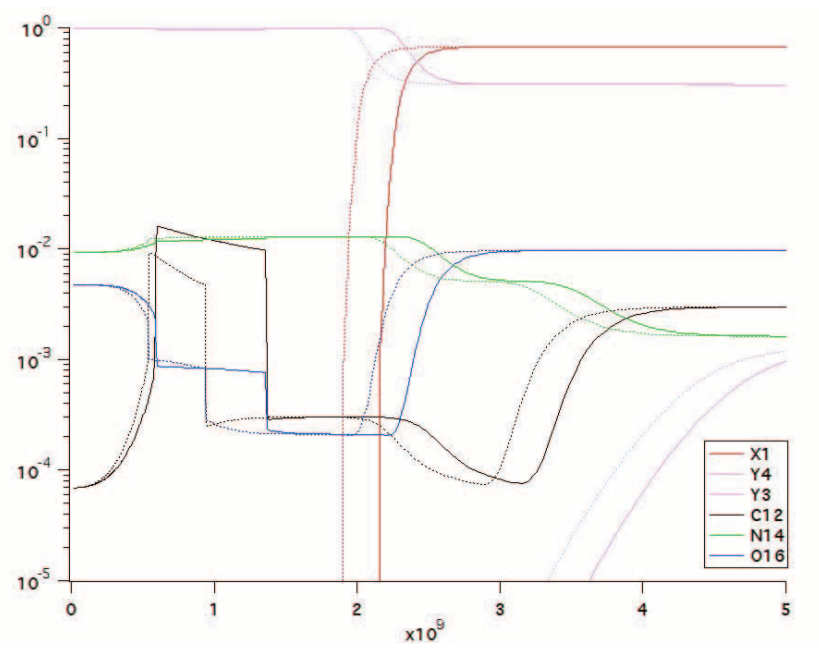

FIG. 7.-Comparison of the composition structure of models E8 and E9, showing the hydrogen-burning shell expanding at about $3.5 \mathrm{~m} \mathrm{~s}^{-1}$. At the peak energy production rate, the convective shell extends from about 0.16 to $0.43 M_{\odot}$, just below the hydrogen-burning shell. 


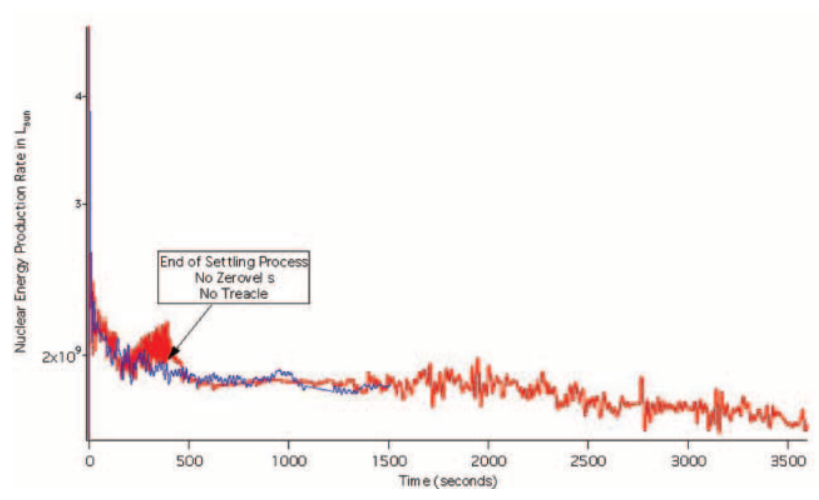

FIG. 8.- Initial transients. In both E9 (red) and E9' (blue), there is an initial transient associated with the onset of convective motion, but this quickly settles to a stable value near that of the 1D model. The more refined mesh, E9', produces a nearly identical result.

convective shell has developed out to $0.43 M_{\odot}$ (from 0.0085 to $\left.0.0195 R_{\odot}\right)$. As discussed in $\S 3.1$, this expansion has caused the energy production from hydrogen burning to drop tremendously.

Because model E9 is very near the peak energy production rate, the start-up transient that occurred while convection established itself was thought to be most likely to result in anomalous behavior (core disruption, core/envelope mixing, etc.). Here the initial energy spike reached nearly $5 \times 10^{9} L_{\odot}$ and again quickly dropped to the expected value (Fig. 8). The initial value of the speed limiter was set to $10 \mathrm{~km} \mathrm{~s}^{-1}$ and was removed after less than $100 \mathrm{~s}$. We again attempted to apply the Zerovel option (between 300 and $400 \mathrm{~s}$ ), but by $400 \mathrm{~s}$ simply left the simulation alone, with no ad hoc options. This resulted in a structure that produced energy from helium burning near $1.8 \times 10^{9} L_{\odot}$ for the hour simulated.

This model was run twice, once with a 0.86 megazone mesh, E9, and again with 1.36 megazones, E9'. The higher resolution (larger mesh) run was started with the same speed limiter, but it was removed after only $50 \mathrm{~s}$. In E9', no attempts were made to use the Zerovel option. Except for the short period when Zerovel was tested in E9, the evolution of the energy production rate was seen to be agreement in the separate simulations (Fig. 8). Both simulations show small fluctuations in the instantaneous energy production rate, as hot spots occur and are quenched by expansion and plume generation. The fluctuations appear to be small in both simulations, but they are slightly smaller in the higher resolution case. The behavior brings into question the overall stability of the lower resolution model against the hydrodynamic fluctuations that lead to hot spots. To test this, a numerical experiment was performed on the lower resolution model, E9. The temperature of 18 contiguous zones was artificially increased from their original values (near $16 \mathrm{keV}$, or $1.9 \times 10^{8} \mathrm{~K}$ ) to a value of $25 \mathrm{keV}$. The peak temperature variation seen in the unperturbed models was less than $100 \mathrm{eV}$, so a $9 \mathrm{keV}$ increase was a tremendous perturbation. Nevertheless, the model demonstrated that it was stable against such fluctuations. After the large temperature perturbation was artificially introduced, there was a spike in the total energy production rate, which increased by a factor of 2 for about $1 \mathrm{~s}$. Following the spike, there was a trace of excess energy production for another $5 \mathrm{~s}$, as a rapidly rising plume was initiated (Fig. 9).

Within $20 \mathrm{~s}$, that plume had risen farther and faster than any of the normal plumes, and the energy production rate had returned to the prehot spot level. This is strong evidence that even the coarser resolution used was stable against the normal tempera-
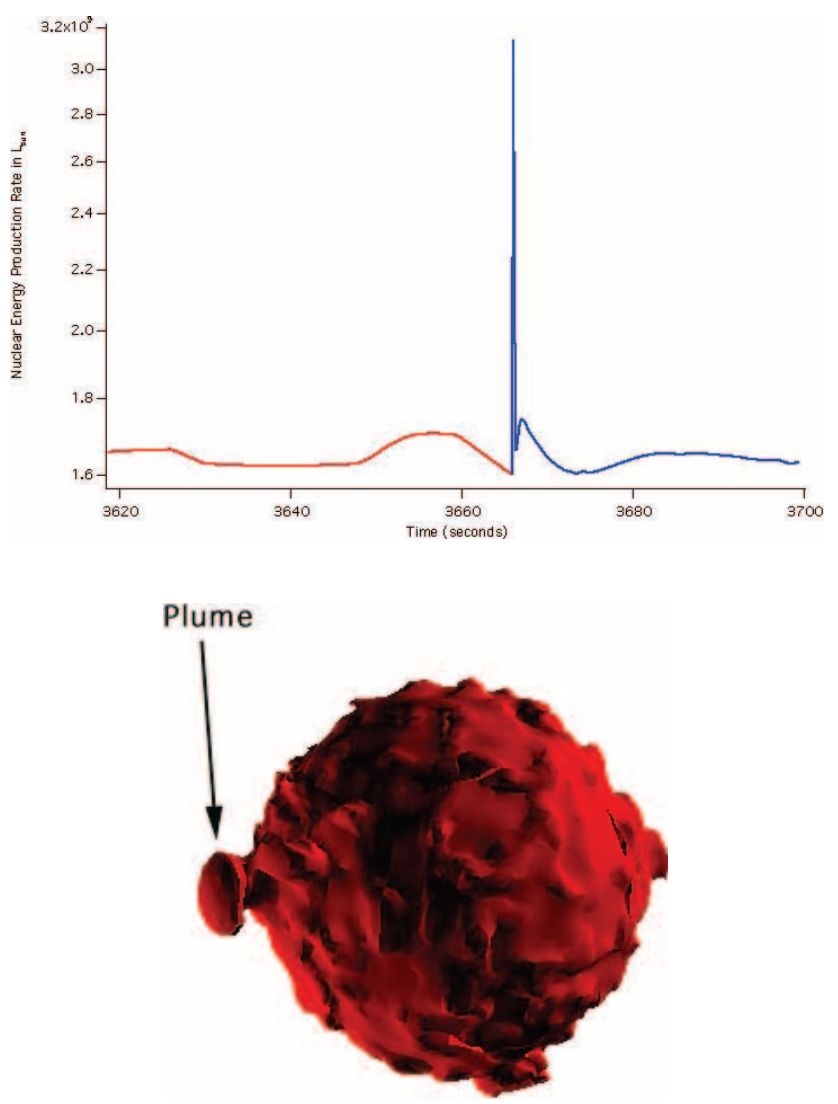

FIG. 9.- Run E9 perturbed. Top, Energy production rate from an artificially introduced hot spot, showing local expansion and plume formation is a robust stabilizing force; bottom, plume forming a mushroom cloud as seen in an ${ }^{18} \mathrm{O}$ contour (constant mass fraction).

ture fluctuations in the convective shell. The $10^{8} L_{\odot}$ model (E8) was also tested for stability against a range of volume and temperature perturbations that far exceeded the natural fluctuations that were seen to occur naturally and again proved stable.

As described above the initial 3D models had no velocity information, and the initial motion occurs as a result of a strong burst of energy production in a thin layer at the bottom of the convective shell of the 1D model. As this layer expands, the energy production rate decreases, but the layer becomes RayleighTaylor unstable. The result is a pattern of rising and falling areas that shows mesh imprinting associated with the small deviations from sphericity. In the $10^{9} L_{\odot}$ model (E9) this patterning persists as plumes rise and settle for over 20 minutes of the hour modeled. The pattern gradually dissipates, and well before the end of this run the plumes appear to occur randomly, with no special connection to the mesh structure.

In Figure 10, a contour is shown with a fixed mass fraction of ${ }^{18} \mathrm{O}$. As the $1 \mathrm{D}$ model did not track this isotope, all of it is produced in the 3D model, and it serves as a tracer of element production and convective distribution in the helium-burning shell. Although some articial mixing is introduced through the occasional Eulerian remapping of the Langrangian mesh, this effect is small compared with the genuine changes of nuclear evolution.

Figure 10 also shows the velocity vectors lying in a plane through the center of the star and a circle showing the limit of convection in the 1D model. In the outer areas, the velocity vectors show a persistent azimuthal ringing that has some mesh pattern in it. This ringing does not result in mixing and makes simple speed tracking undependable as an indicator of the 


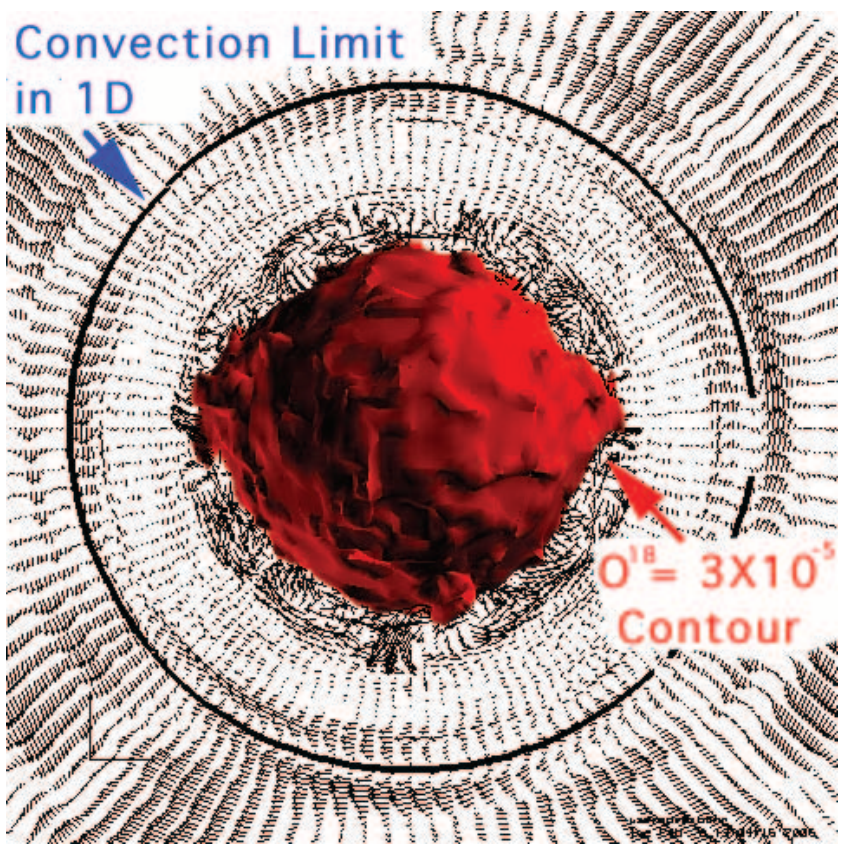

FIG. 10.-For run E9, the outer 1D convective limit, shown on a slice through the center of the star. That plane also shows the local velocity vectors. Superimposed on this slice is a $3 \mathrm{D}$ contour of fixed ${ }^{18} \mathrm{O}$ mass fraction.

operation of convection. In the neighborhood of the ${ }^{18} \mathrm{O}$ surface shown, the velocity vectors show a very different behavior. Here they show patterns of rising and falling regions as plumes develop, cool, mix, and fall. Over the hour followed in model E9, substantial mixing occurred over the inner half of the expected region and was slowly moving outward.

An alternative illustration of this mixing is shown in Figure 11, a color plot showing the distribution of ${ }^{18} \mathrm{O}$ on a slice through the star (run E9). The original convective shell is defined by ${ }^{12} \mathrm{C}$ contours where this isotope has a mass fraction of 0.005 (yellow). The ${ }^{18} \mathrm{O}$ is created in hot spots in a thin layer at the bottom of this convective region and mixed both outwardly and inwardly. The peak ${ }^{18} \mathrm{O}$ mass fraction is near $5 \times 10^{-5}$, and a contour of $10^{-8}$ (red) is shown as an indication of the maximum extent of the mixing. Again, in the first hour of simulation, the mixing has worked its way through approximately half the region expected from the

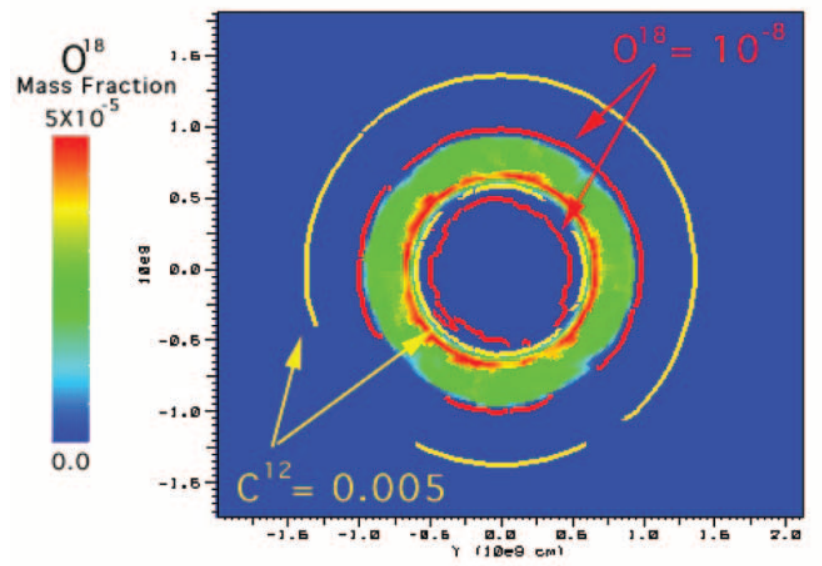

FIG. 11.- Slice through the center of the star during run E9. The color shows the mass fraction of ${ }^{18} \mathrm{O}$. Also shown are yellow and red contour lines for specific mass fractions of ${ }^{12} \mathrm{C}$ and ${ }^{18} \mathrm{O}$.

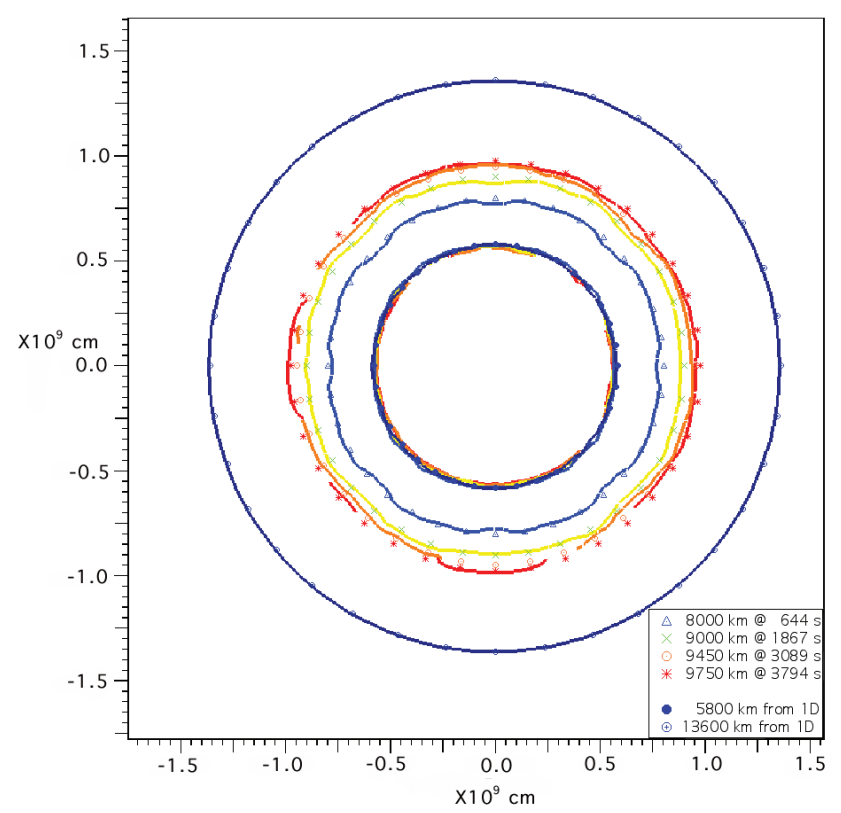

FIG. 12.- Inner and outer dark blue circles, showing the extent of convection in the $1 \mathrm{D}$ model. The intermediate near-circles in pale blue (with triangles), green (with crosses), orange (with circles), and red (with asterisks) show the approximate outer limits of convection in the 3D model (run E9), at times $644,1867,3089$, and $3794 \mathrm{~s}$. This radius expands roughly as $t^{1 / 2}$ and can be expected to catch up with the $1 \mathrm{D}$ boundary in $\sim 5 \mathrm{hr}$.

1D model. ${ }^{18} \mathrm{O}$ also appears to be working its way into the nonburning portion of the helium core. Some of this may result from local captures of helium on ${ }^{14} \mathrm{~N}$ nuclei, but the irregularity of the surface argues that downwardly convective overshoot is occurring at the base of the convective region.

Normal stellar evolution is governed by the slow composition change resulting from nuclear reactions, but the structure change at the peak of the helium flash is a thermal timescale adjustment. As energy is produced, the convective portion of the core is transformed by expansion from a degenerate configuration supported by Fermi pressure toward a Maxwell-Boltzmann gas. The thin region that separates the outer convective shell from the hydrogen-burning shell is pushed slowly outward and also decompressed as the gravity is lowered. The effect of this on the hydrogen-burning shell is most clearly manifested in the sharp drop in the energy production rate from hydrogen burning.

Figure 12 shows another way of illustrating the growth of convection in the helium-burning shell (run E9). The large and small dark blue circles give the limits of convective mixing in the original 1D model. Pale blue, green, orange, and red circles indicate roughly the limits of convective mixing (according to the ${ }^{18} \mathrm{O}$ contour) at four epochs ranging from 644 to $3974 \mathrm{~s}$. The inner boundary is almost independent of time, but shows a very slight tendency to "downward overshoot." The outer boundary moves outward and can be approximately represented by $r \propto t^{1 / 2}$. We can expect that it may reach roughly the neighborhood of the $1 \mathrm{D}$ boundary in about $5 \mathrm{hr}$.

As the energy production rates agree reasonably well between the $1 \mathrm{D}$ and 3D simulations, it is no surprise that the core expansion rates are similar. Over the course of an hour of simulation, the helium core (defined from the hydrogen composition profile) of run E9 expanded at a rate of $18 \mathrm{~m} \mathrm{~s}^{-1}$ in the 3D simulation. Beginning with the same model, the 1D helium core of the 1D models expanded at a rate of $13 \mathrm{~m} \mathrm{~s}^{-1}$ over a $4 \mathrm{hr}$ period. While 


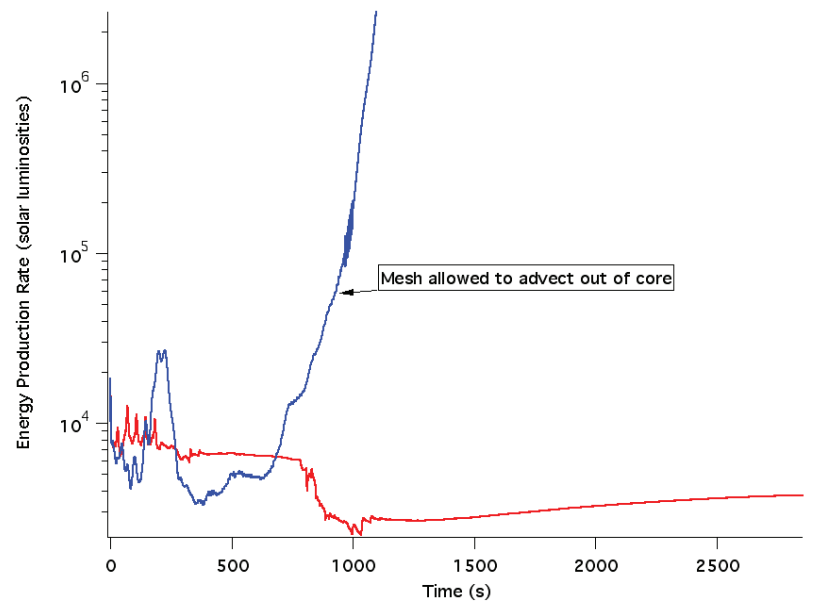

FIG. 13.- Inadequate resolution results in greater fluctuation in the energy production rate and a nuclear runaway.

the expansion rate is slightly faster in the $3 \mathrm{D}$ simulation, the speed is far below the local sound speed, and hydrostatic modeling should capture most of the behavior of the helium flash.

Before turning to a discussion of these results, we mention a final numerical experiment related to the stability of helium flash simulations. This experiment was preformed on the lowest luminosity model (E4) with the lowest mesh resolution. A short simulation was done with E4 in which the mesh was not so concentrated in the helium-burning convective shell. Furthermore, the ALE option was set to allow the mesh to slowly move into the outer regions. The result of these choices is shown in Figure 13.

As discussed in the comparison of the energy production rate for models $\mathrm{E} 9$ and $\mathrm{E} 9^{\prime}$, higher resolution appears to reduce the rate fluctuations associated with hot spots at the base of the convection zone. Sufficient reduction in resolution results in a nuclear runaway in which $100 \mathrm{yr}$ of energy production rate increase occurs in 10 minutes. Various tests done here demonstrate that all of our models were sufficiently resolved to eliminate this gross instability. We believe this confirms that 3D modeling, given sufficient resolution, is able to produce convective motion that is adequate to carry the heat flux and does not need to be supported (or opposed) by approximate modeling of small-scale turbulence.

Figure 14 shows the radial velocity color coded so that red is outward and blue is inward. The heavy narrow blue ring is the hydrogen-burning shell. It can be seen that there is possibly significant motion of an apparently convective nature outside this shell. It is not clear what this is due to, but it is difficult to see why this should be only a response to helium ignition. If it is real, it may indicate a kind of motion that might take place above the hydrogen shell even if there is no helium flash. Astrophysicists have frequently noted (e.g., Ivans et al. 2001; Cavallo \& Nagar 2000) that some giants, not necessarily beyond the helium flash, have anomalous surface abundances that suggest the possibility of mixing between the convective envelope and the hydrogenburning shell. We appear to be seeing some motion that might, if it occurs in a preflash red giant, persist and grow so as to allow some mixing of hydrogen-burning products out to the convective envelope.

\section{DISCUSSION}

1. The 3D simulations were robustly stable, and, apart from the convective shell itself, the behavior of the star was consistent

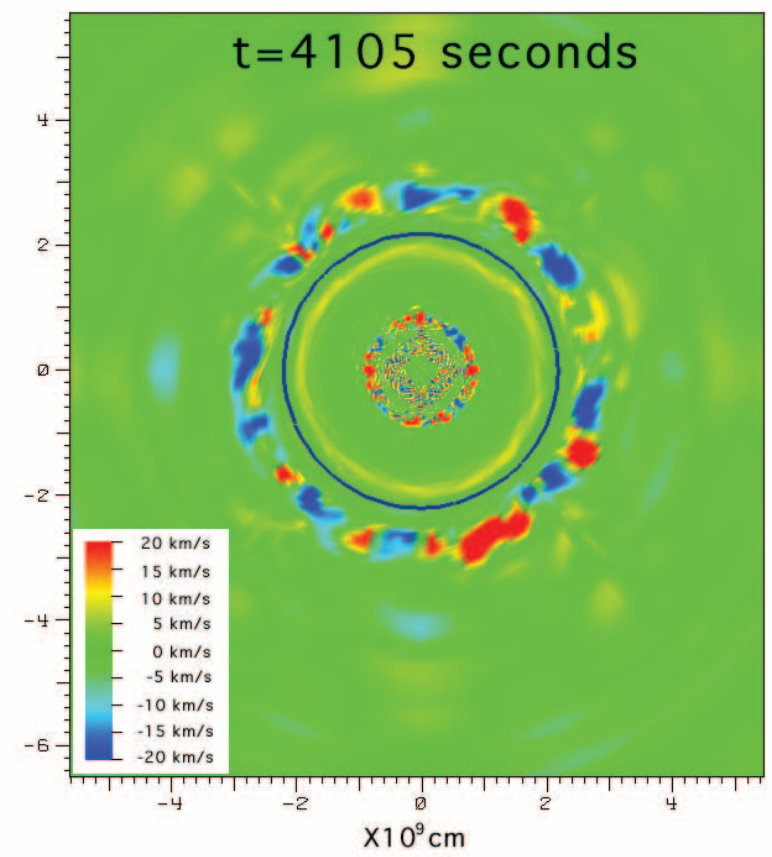

FIG. 14.-Convective and other motion in run E9, about $4000 \mathrm{~s}$ after the start. The color-coded variable is the radial velocity, with red outward and blue inward. The hydrogen-burning shell is marked by a heavy narrow blue ring. Some motion is visible outside the H-shell, in addition to the He-driven convection well inside it.

with hydrostatic modeling, even at the peak of the helium flash. Although exact spherical symmetry is obviously required in the $1 \mathrm{D}$ code, the $3 \mathrm{D}$ models seem to retain more or less spherical symmetry: there is no tendency for one hot spot to erupt and then dominate the shell, rendering it very asymmetric.

2. Convection is a critical element in determining the evolution through the helium flash. In 1D hydrostatic modeling, convection is an approximation with effectively no information on the complex process by which hot spots develop and relax themselves.

3. In all of our models, the convection approached but never exceeded the outer boundary of convection as determined from a stability criterion in the 1D code. However, we cannot claim that overshoot does not occur in longer runs. In 1D, the inner boundary of the convective shell is nearly coincident with the peak-energy-producing shell. Our simulations do show a slight and potentially significant mixing below the convective shell from downward overshoot. This leads to erosion of the nonburning central core, and if it continues, it could reduce or eliminate the miniflashes that occurred in the $1 \mathrm{D}$ simulation. We intend to explore this further.

4. In the future, we intend to address all of the following: rotation, magnetic fields, and low metallicity. We shall also pursue further the possibility that some slow mixing outside the hydrogen-burning shell during first giant branch evolution might affect the surface abundances.

This study has been carried out under the auspices of the US Department of Energy, National Nuclear Security Administration, by the University of California, Lawrence Livermore National Laboratory, under contract W-7405-Eng-48. J. C. L. was partially supported by the Australian Research Council. We are indebted to R. Palasek for assistance with the graphics. 
Alexander, D. R., \& Ferguson, J. W. 1994, ApJ, 437, 879

Anderson R. W., Elliott, N. S., \& Pember, R. B. 2004, J. Comput. Phys., 199, 598

Bazán, G., Cavallo, R. M., Dearborn, D. S. P., Eggleton, P. P., Dossa, D. D., Keller, S. C., Taylor, A. G., \& Turcotte, S. 2003, in ASP Conf. Ser. 293, 3D Stellar Evolution, ed. S. Turcotte et al. (San Francisco: ASP), 1

Cavallo, R. M., \& Nagar, N. M. 2000, AJ, 120, 1364

Dearborn, D. S. P., Wilson, J., \& Mathews, G. 2005, ApJ, 630, 309

Deupree, R. G. 1996, ApJ, 471, 377

Eggleton, P. P. 1971, MNRAS, 151, 351

Eggleton, P. P., Faulkner, J., \& Flannery, B. P. 1973, A\&A, 23, 325

Faulkner, J. 1966, ApJ, 144, 978

Graboske, H. C., Dewitt, H. E., Grossman, A. S., \& Cooper, M. S. 1973, ApJ, 181,457

Henyey, L. G., Wilets, L., Böhm, K. H., Lelevier, R., \& Levee, R. D. 1959, ApJ, 129, 628

\section{REFERENCES}

Hubbard, W. B., \& Lampe, M. 1969, ApJS, 18, 297

Itoh, N., Adachi, T., Nakagawa, M., Kohyama, Y., \& Munakata, H. 1989, ApJ, 339,354

Itoh, N., Mitake, S., Iyetomi, H., \& Ichimaru, S. 1983, ApJ, 273, 774

Itoh, N., Mutoh, H., Hikita, A., \& Kohyama, Y. 1992, ApJ, 395, 622

Ivans, I. I., Kraft, R. P., Sneden, C., Smith, G. H., Rich, R. M., \& Shetrone, M. 2001, AJ, 122, 1438

Mestel, L. 1952, MNRAS, 112, 583

Pols, O. R., Tout, C. A., Eggleton, P. P., \& Han, Z. 1995, MNRAS, 274, 964

Rogers, F. J., \& Iglesias, C. A. 1992, ApJ, 401, 361

Schwarzschild, M., \& Härm, R. 1962, ApJ, 136, 158

Timmes, F. X., Hoffman, R. A., \& Woosley, S. E. 2000, ApJS, 129, 377

Woodward P., \& Colella P. 1984, J. Comput. Phys., 54, 115 